\title{
Correlation Analysis of Roughness Surface Height Distribution Parameters and Maximum Mises Stress
}

Duo Yang ( $\sim$ duoyoung688@163.com )

Central South University

\section{Qibo Wang}

Central South University

Jinyuan Tang

Central South University

\section{Fujia Xia}

Central South University

\section{Wei Zhou}

Hunan University of Science and Technology

\section{Yuqin Wen}

Central South University

\section{Research Article}

Keywords: Roughness surface, Height distribution, Contact stress, Regression analysis, Particle swarm algorithm

Posted Date: September 23rd, 2021

DOl: https://doi.org/10.21203/rs.3.rs-898126/v1

License: (9) This work is licensed under a Creative Commons Attribution 4.0 International License. Read Full License

Version of Record: A version of this preprint was published at Surface Topography: Metrology and Properties on March 1st, 2022. See the published version at https://doi.org/10.1088/2051-672X/ac5d6b. 


\title{
Correlation Analysis of Roughness Surface Height Distribution Parameters and Maximum Mises Stress
}

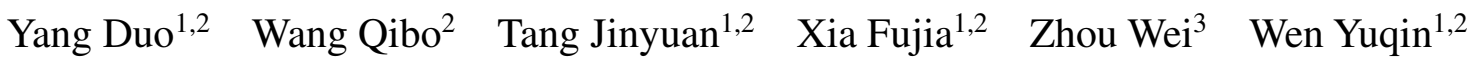

\begin{abstract}
Roughness surfaces contact analysis is an advanced research topic in interface design. The 3D rough surface amplitude

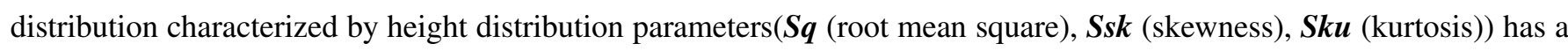
great influence on the extreme value and distribution of the interface contact stress. However, the relationship between height distribution parameters and surface maximum mises stress $\left(\boldsymbol{\sigma}_{\max }\right)$ is still unclear and lacks of in-depth study. With the assistance of roughness surface reconstruction and contact stress algorithm proposed by the research group, $\sigma_{\max }$ under a large sample was calculated and used as the data support for correlation analysis. Through BP neural network, global sensitivity qualitative (Morris) and quantitative (Sobol) analysis methods, the relationship between $\boldsymbol{S q}, \boldsymbol{S s k}, \boldsymbol{S k u}$ and $\boldsymbol{\sigma}_{\max }$ under different loads is studied. Based on complete polynomial and permutation combination method, the optimal correlation model between height distribution parameters and $\boldsymbol{\sigma}_{\boldsymbol{m a x}}$ was established, and particle swarm algorithm was introduced to analyze $\sigma_{\max }$ extreme values under different $\boldsymbol{S} \boldsymbol{q}$. The results show that: (1) Under different loads, the order about height distribution parameters influence on surface contact stress is: $\boldsymbol{S q}>\boldsymbol{S s} \boldsymbol{k}>\boldsymbol{S k \boldsymbol { u }}$, and as the load increases, the influence of $\boldsymbol{S s} \boldsymbol{k}$ and $\boldsymbol{S k} \boldsymbol{u}$ gradually decreases. (2) In different roughness surfaces, the influence of $\boldsymbol{S} \boldsymbol{s} \boldsymbol{k}$ and $\boldsymbol{S k \boldsymbol { u }}$ on the contact performance is significantly full of discrepancy. The research results provide reference and technical support for active design of rough surface microstructure to improved contact performance.
\end{abstract}

Key Words: Roughness surface; Height distribution; Contact stress; Regression analysis; Particle swarm algorithm

\footnotetext{
${ }^{1}$ State Key Laboratory of High Performance Complex Manufacturing, Central South University, Changsha, Hunan, China

${ }^{2}$ The College of Mechanical and Electrical Engineering, Central South University, Changsha, Hunan, China

${ }^{3}$ Hunan Provincial Key Laboratory of High Efficiency and Precision Machining of Difficult-to-Cut Material, Hunan University of Science and Technology, Xiangtan, China;

Corresponding author

${ }^{1}$ State Key Laboratory of High Performance Complex Manufacturing, Central South University, Changsha, Hunan, China

E-mail: jytangcsu_312@163.com
} 


\section{Introduction}

Surface morphology significantly affects the contact, wear and friction properties of the components, which restricts its service performance and working hours[1-3]. In order to describe surface topography more accurately, midline characterization method based on the profile section was proposed. The method mainly used surface height arithmetic square root $\boldsymbol{R} \boldsymbol{a}$ to evaluate the surface quality, and had been widely applied in many industries[4, 5]. But with the development of science and technology, the researchers found that only a single roughness parameter characterizing topography can't contain all the surface information. Therefore, roughness parameters based on the characteristics of surface peaks and valleys and Abbott curve: $\boldsymbol{R} \boldsymbol{p}$ (maximum profile peak height), $\boldsymbol{R} \boldsymbol{v}$ (maximum profile valley depth), $\boldsymbol{R} \boldsymbol{m} \boldsymbol{r}$ (relative material ratio) were used to distinguish surface extremum characteristic and bearing performance[6-8]. In view of the convenience about roughness parameters, researchers from all over the world defined a series of different roughness parameters according to the actual needs, but ignored the rigor of parameter definition, resulting in the continuous increase of parameters and "parameter explosion" phenomenon[9].

The redundancy of parameters and the disparate definition of symbols increased the obstacles to surface topography characterization, so the International Standards Organization Technical Committee formulated roughness standard draft ISO 4287 in 1997 to unify parameter names and symbols, and further divide them into amplitude parameters, spacing parameters, hybrid parameters, and material ratio curves and related parameters[10, 11]from parameter definitions. However, two-dimensional profile section method was powerless to avoid the loss of topography spatial information and difficult to meet the requirements of modern precision machining. In contrast, three-dimensional surface roughness parameters, widely used in later work, can reflect topography characteristics more comprehensively and truly. The principle of parameter definition is to collect height data in a designated area and store it as a height matrix, and obtain information such as surface height distribution and spatial distribution based on the midplane of the topography $[4,12]$. Although the threedimensional parameter classification standard covers functions and related parameters, they are generally used to evaluate the oil storage and lubrication performance of the surface, which cannot be combined with contact performance[13].

It is well known that height distribution parameters are directly related to surface roughness, which significantly affects interface contact strength and fatigue performance of the workpiece. In addition, under the premise of a given autocorrelation function, height distribution parameters are considered to carry surface microstructure statistical characteristics, determined by the manufacturing principle and processing parameters, which can well characterize the surface topography[14, 15]. For example, under different roughness conditions, Tayebi et al. firstly studied the influence of skewness and kurtosis in Gaussian distribution surface on the static friction coefficient[16]. On the basis, Sedlaček et al. set the same root mean square value to observe and analyze the correlation law between skewness, kurtosis and surface dynamic friction coefficient in the contact surface to clarify height distribution parameters influence on the surface friction performance[17, 18]. However, Yan et al. did a research on the effects of skewness and kurtosis on fatigue life under point contact of mixed elastohydrodynamic lubrication[19]. To reveal the mapping relationship between height statistical distribution parameters and friction and wear properties, Zhu et al. explained height distribution parameters influence mechanism about the friction and wear by studying the correlation between height distribution parameters and surface bearing area ratio and establishing the formula between bearing area ratio and friction coefficient in dry friction and lubrication friction[20]. In terms of correlation analysis between contact performance and roughness, Yang et al. analyzed the influence of roughness parameters on contact performance with the help of statistical correlation theory, neural network and sensitivity analysis[21, 22]. However, the above researches were either based on experiments, whose samples were insufficient and hard to obtain, or did not take height distribution parameters as research objects. Therefore, the related work generally focused on qualitative analysis, leading to limitations in conclusions. And the correlation between height distribution parameters and contact properties of rough surfaces has been obscured by fog.

Since surface reconstruction algorithm can provide large sample in a short time and the default surface morphology characteristics, it is an excellent idea to apply the algorithm to study the relationship between surface properties and performance. And height distribution parameters were the key parameters in the surface reconstruction algorithm, which means that under the premise of a given autocorrelation length and texture direction, a surface with different morphological characteristics can be generated by setting the $\boldsymbol{S} \boldsymbol{q}, \boldsymbol{S} \boldsymbol{s}, \boldsymbol{S} \boldsymbol{k} \boldsymbol{u}[23,24]$. Secondly, surface maximum mises stress $\left(\boldsymbol{\sigma}_{\max }\right)$ directly reflects the surface bearing capacity and can be well used to evaluate the surface contact performance. As a result, it is wise to clarify the influence of the surface 
topography on the contact performance by illustrating the relationship between height distribution parameters and $\sigma_{\text {max }}$.

In the first place, based on the surface reconstruction algorithm proposed by Liao et al. in literature [23], 264 surfaces with different characteristics were generated in this paper, meanwhile surface maximum mises stress $\left(\sigma_{\max }\right)$ under different loads was obtained from Wen's et al three-dimensional rough surfaces contact analysis model[25], which provided data support for the correlation discussion.

Followed by the first step, considering that there is no direct theoretical analysis model between height distribution parameters and stress, BP neural network was introduced to build a mapping model between them, and the global sensitivity analysis method was used to evaluate the influence of height distribution parameters on contact stress.

Finally, optimal correlation model between height distribution statistical parameters and maximum mises stress was constructed through complete polynomials and combination ideas. The surface contact performance difference under different $\boldsymbol{S q}$ was demonstrated by particle swarm algorithm. The research quantifies the influence of height distribution parameters on the contact stress under different loads, and provides a reference and technical support for the active design of rough surface microstructures to improve components service performance.

\section{Data and methods}

\subsection{Data Sources}

On account of the truth that the research focuses on the use of data analysis to dig out the correlation law between height distribution parameters and rough surface contact stress, only a brief introduction to the data source is given. The detailed calculation process of surface reconstruction algorithm and contact model can be found in the literature [23] and [25].

(1) Reconstruction surface

Surface reconstruction algorithm proposed in Literature [23] is used to randomly allocate height distribution parameter values by MATLAB random function under the premise of a given autocorrelation length of 32. The parameter settings are shown in the Table 1.

Table 1. Parameter Settings

\begin{tabular}{cccc}
\hline \multirow{2}{*}{ Range } & \multicolumn{3}{c}{ Parameter } \\
\cline { 2 - 4 } & $\boldsymbol{S q}$ & $\boldsymbol{S s k}$ & $\boldsymbol{S k u}$ \\
\hline Minimum & 0.1 & -3 & 2.9 \\
Maximum & 0.5 & 1 & 10 \\
\hline
\end{tabular}

The sampling interval is set as $1 \mu \mathrm{m}$, and the total area $\boldsymbol{A}=480 \times 640=0.307 \mathrm{~mm}^{2}$, and finally 264 sets of non-Gaussian reconstruction rough surfaces are generated. Fig.1 shows two reconstruction surfaces with different roughness.

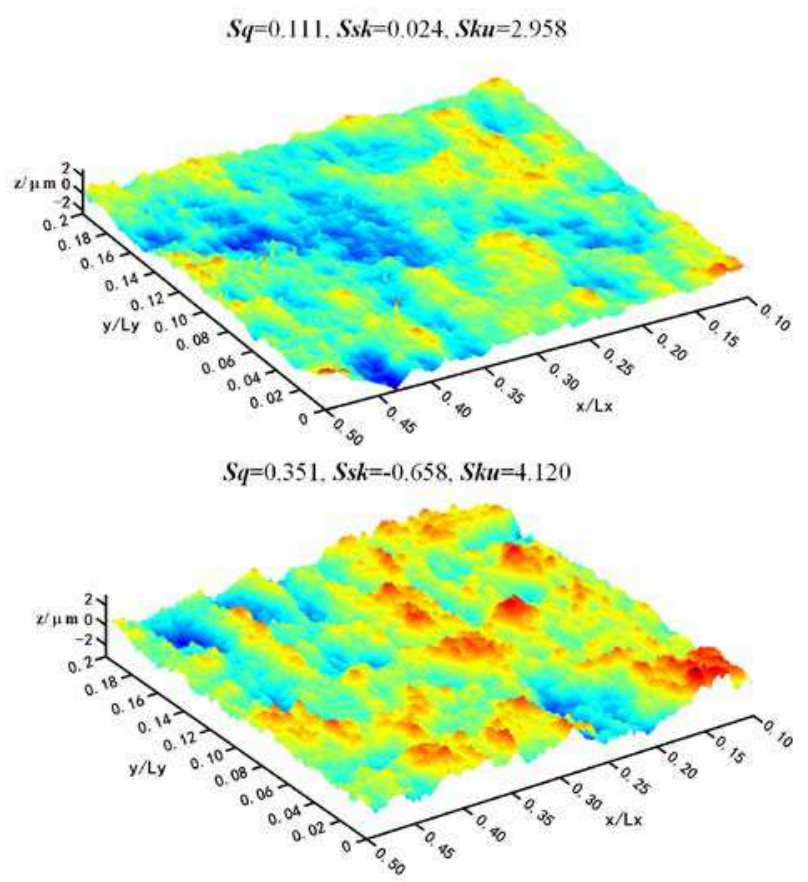

Figure.1 Reconstruction Surface Topography

(2) Surface contact stress

Having 264 reconstruction surfaces been obtained, it is no sweat to calculate $\boldsymbol{\sigma}_{\max }$ from Wen's elasticplastic contact for three-dimensional rough surfaces considering interaction of asperities. Mechanical property parameters of the surface are set as shown in Table 2.

Table 2. Surface Mechanical Property

\begin{tabular}{cl}
\hline Name & Value \\
\hline Elastic Modulus & $209 \mathrm{GPa}$ \\
Poisson's ratio & 0.29 \\
Yield strength & $850 \mathrm{MPa}$ \\
Hardness & $2.38 \mathrm{GPa}$ \\
\hline
\end{tabular}

In order to analyze the influence of the surface amplitude characteristics in different loads, dimensionless loads are set to 0.0002, 0.0005, 0.001, and the ratio is $1: 2.5: 5$. Finally, the working conditions based on the three loads are regarded as light load, medium load and heavy load, respectively. The conversion formula between dimensionless load and actual load is as follows:

$$
\boldsymbol{P}_{n}=\frac{\boldsymbol{P}_{t}}{\boldsymbol{A}_{n} * \bar{E}}, \overline{\boldsymbol{E}}=\frac{\boldsymbol{E}}{\left(1-\boldsymbol{v}^{2}\right)^{2}}
$$

$\boldsymbol{P}_{\boldsymbol{t}}$ is the actual load on the surface, $\boldsymbol{P}_{\boldsymbol{n}}$ is the dimensionless load; 
$\bar{E}$ is the equivalent elastic modulus;

$\boldsymbol{A}_{n}$ is the nominal contact area;

The surface is not set to be in full-area contact. because of the usual roll-slip condition in interface contact. $A_{n}$ is $200 \times 640=0.128 \mathrm{~mm}^{2}$ in the paper. According to formula (1), actual loads are $2.8929 \mathrm{~N}, 7.2322 \mathrm{~N}$ and $14.4645 \mathrm{~N}$. And the surface maximum mises stress of three loads are recorded as mises 1, mises 2 and mises 3 in respective. The sample distribution of 264 surface mises stress under different loads is shown in Box Fig.2.

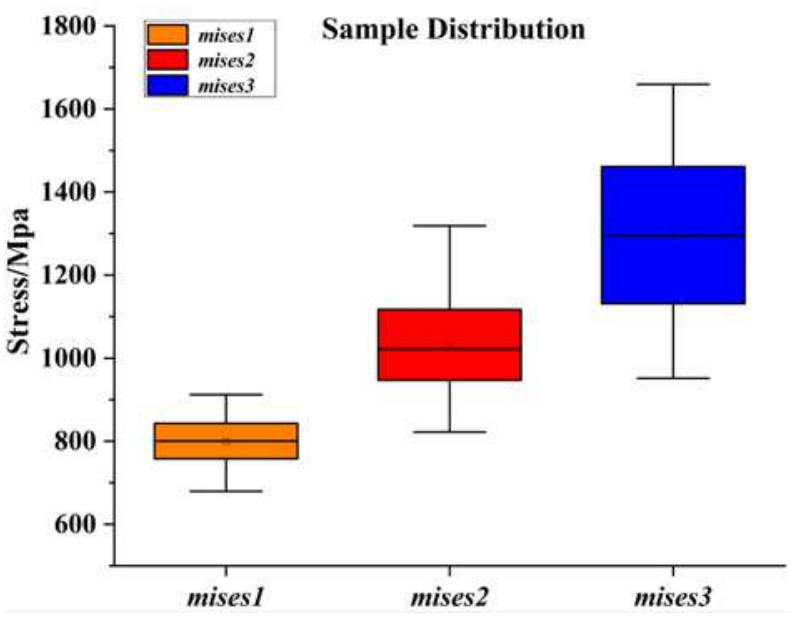

Figure.2 Stress sample distribution

According to Fig.2, most of the surfaces under mises 1 don't reach the yield strength of the material, and the surfaces are in the elastic deformation stage. Although most of surfaces in mises 2 exceeds the yield strength, the excess amplitude is low and part of them is still in the elastic deformation stage. In mises3, all the surfaces exceeds the yield strength and enter the plastic deformation stage.

It is well known that when considering the surface micro-morphology, the surface plastic deformation is easy to occur in interface contact. The strength criterion cannot be used to simply judge the surface failure. Therefore, stress samples distribution characteristics in Fig. 2 can clarify the phenomenon that the failure risk of the surface increases as the load increases, which is in line with the preset light and heavy load conditions.

\subsection{Global sensitivity analysis}

(1) Sensitivity analysis model

There is no perfect theoretical analysis model between height distribution parameters and surface contact stress as the research object at present. And the mapping relationship between them is bound to be necessarily nonlinear, so BP neural network is chosen as the sensitivity analysis model.

The structure of BP neural network is composed of input layer, hidden layer and output layer with connecting lines between different layers to ensure the transmission of data. Each connecting line will have a separate weight value to constitute the weight matrix in BP neural network. Based on the weight matrix, the connection strength between neural units in different layers can be quantitatively evaluated. Usually, in complex time series or computer vision data sets, BP neural network will choose multi-layer hiding layer to increase model reliability. However, multi-layer hidden layer is easy to lead to problems such as overfitting and computation speed reduction. Therefore, in general simple data sets, two or more hidden layers are rarely used[26]. Owing to the data characteristics from observation, a single hidden layer network model is constructed. Fig. 3 shows BP model structure.

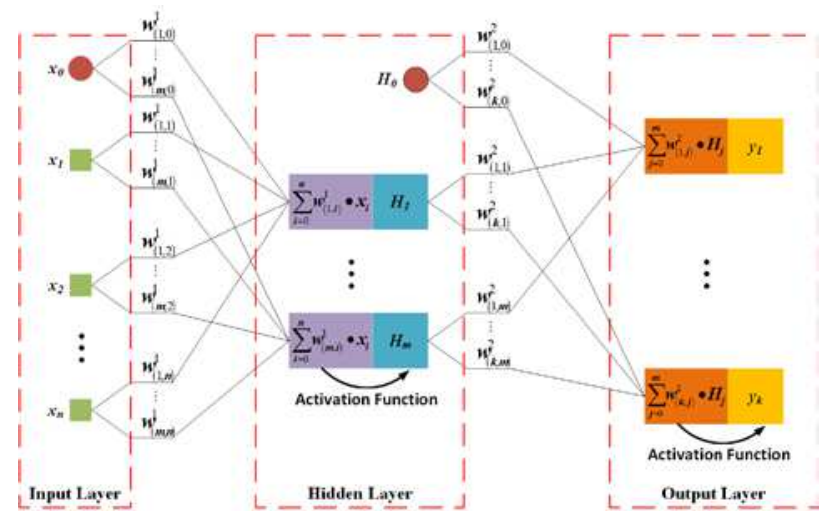

Figure.3 BP neural network

The input layer is $\boldsymbol{S q}, \boldsymbol{S s} \boldsymbol{k}$ and $\boldsymbol{S k} \boldsymbol{u}$, and the output layer is mises1, mises2 and mises3. After training, the mapping model between height distribution parameters and $\sigma_{\max }$ in multiple loads can be obtained.

(2) Principle of sensitivity analysis

BP neural network provides a reliable model for global sensitivity analysis. Global sensitivity analysis method qualitatively and quantitatively evaluates the influence of different height distribution parameters on $\sigma_{\max }$ by calculating sensitivity coefficient. The relevant principle is as follows:

\section{(1) Morris method}

Morris sensitivity analysis is a qualitative global sensitivity analysis. The method advantage is that it is stable in sensitivity coefficient calculation, and new samples can be generated from origin samples to conduct research, but insufficient to capture sample features[27], so the method is generally used to preliminarily determine the relative sensitivity at the first step. The analysis process is in display.

(1) Morris new samples are randomly generated, and its core formula is as follows:

$$
B^{1}=J_{1} x^{*}+\frac{m}{2}\left[\left(2 B-J_{m}\right) D^{*}+J_{m}\right]
$$

$\boldsymbol{B}^{1}$ represents $(k+1) \times k$ order Morris new sample matrix , where $k$ is the number of variables, and there is only a 
single parameter difference between two adjacent rows in the matrix with the rest exactly in the same;

$\boldsymbol{J}_{\mathbf{1}}$ is the unit column vector of order $\boldsymbol{k}+1$;

$\boldsymbol{x}^{*}$ is a randomly generated parametric base sample $\left(\boldsymbol{x}_{\boldsymbol{l}}\right.$, $\left.x_{2}, \ldots x_{i}, \ldots, x_{k}\right)$, belonging to the core in formula(2). Here is its source description. After setting level parameter $\boldsymbol{p}, \boldsymbol{x}_{\boldsymbol{i}}$ becomes a discrete and normalized parameter set $\boldsymbol{M}(0,1 /(\boldsymbol{p}-1), 2 /(\boldsymbol{p}-2), \ldots, 1)$. And then $\boldsymbol{x}^{*}$ will be born by means of randomly selecting the element from set $\boldsymbol{M}$ in turn;

$\boldsymbol{m}$ is the disturbance factor;

$\boldsymbol{B}$ is the $(k+1) \times k$ order matrix, for any element in $\boldsymbol{B}$ : $b_{i j}=\left\{\begin{array}{ll}1 & j \leq i \\ 0 & j>i\end{array} ;\right.$

$\boldsymbol{J}_{\boldsymbol{m}}$ is the $(k+1) \times k$ order matrix with all positions filled with 1 ;

$\boldsymbol{D}^{*}$ is a diagonal square matrix of order $\boldsymbol{k}$, and diagonal element is randomly set to \pm 1 ;

(2) Calculate $\boldsymbol{x}_{j}$ Morris sensitivity coefficient in Morris new sample $\boldsymbol{B}^{\mathbf{1}}$. The impact factor $\boldsymbol{p}_{j}(1)$ is as follows:

$$
p_{j}(1)=\frac{f\left(x_{1}, \cdots, x_{j}+m, \cdots, x_{k}\right)-f\left(x_{1}, \cdots, x_{j}, \cdots, x_{k}\right)}{m} \text { (3). }
$$

(3) Repeat the above process $\boldsymbol{n}$ times, then the final Morris sensitivity coefficient of the $j$-th parameter is the average value:

$$
p_{j}=\sum_{l=1}^{n} \frac{p_{j}(l)}{n}
$$

However, the method may cause some $\boldsymbol{p}_{j}(\boldsymbol{l})$ values to have positive and negative offset behaviors, resulting in parameters true influence unable to be observed. Therefore, Campolongo et al[28]proposed an improved Morris sensitivity coefficient calculation formula.

$$
p_{j}=\sum_{l=1}^{n} \frac{\left|p_{j}(l)\right|}{n}
$$

The improved method is adopted in the paper.

\section{(2) Sobol method}

Sobol method[29, 30] is a quantitative sensitivity analysis based on variance decomposition, which can effectively quantize the influence of a single parameter and parameters interaction in a high-dimensional nonlinear model. So it can well explain the comprehensive influence of a single independent variable on the dependent variable in the case of multiple independent variables. The sample feature capture ability is better than that of Morris method, and thus the sensitive results in large samples are more reliable. The calculation process is as follows:

First, $y=f(X)=f\left(x_{1}, x_{2}, \cdots, x_{k}\right)$ is decompos-ed into multiple sub-models:

$$
\begin{aligned}
f(X)= & f_{0}+\sum_{i=1}^{k} f_{i}\left(x_{i}\right)+\sum_{1 \leq i<j \leq k} f_{i j}\left(x_{i}, x_{j}\right)+ \\
& \cdots+f_{1,2, \cdots, k}\left(x_{1}, x_{2}, \cdots, x_{k}\right)
\end{aligned}
$$

$f_{0}$ is a constant term. According to variance analysis, total variance of $f(X)$ is the sum of the variances of the sub-models:

$$
V=\sum_{i=1}^{k} V_{i}+\sum_{1 \leq i<j \leq k} V_{i j}+\cdots+V_{1,2, \cdots, k}
$$

Where $\boldsymbol{V}$ is the total variance, $\boldsymbol{V}_{\boldsymbol{i}}$ is the single-factor variance, $\boldsymbol{V}_{i j}$ is the two-factor interaction variance, $V_{l, 2, \ldots, k}$ is all the factors interaction variance. After dividing the two ends of formula (7) by $\boldsymbol{V}$, Sobol sensitivity coefficients of different parameters can be obtained.

$$
\sum_{i=1}^{k} S_{i}+\sum_{1 \leq i<j \leq k} S_{i j}+\cdots+S_{1,2, \cdots, k}=1
$$

$S_{i}$ is the first-order sensitivity coefficient of parameter $i$

$S_{i j}$ is the second-order interaction sensitivity coefficient of parameters $\boldsymbol{i}$ and $\boldsymbol{j}$;

$S_{1,2, \ldots, k}$ is the interaction sensitivity coefficient of all parameters;

Through Equation (7), it is without an effort to calculate different orders sensitivity coefficient including parameter $\boldsymbol{i}$. The formula is as follows:

$$
S_{T_{i}}=\sum S(i)
$$

$\boldsymbol{S}(\boldsymbol{i})$ is the different order item of containing parameter $i$. The total effect sensitivity coefficient $S_{T_{i}}$ is used to evaluate the comprehensive influence of different height distribution parameters.

\subsection{Complete polynomial}

Although BP neural network can express the mapping relationship between variables in a fantastic way, the excessive connection lines and complex activation functions restrict its application in regression analysis. Therefore, to face the double in finding the explicit general expression and optimal correlation model between height distribution parameters and $\sigma_{\max }$, a complete polynomial nonlinear model is introduced to step through the pit. Here's how it works[31, 32].

The target response function is expressed as a complete polynomial combination of $\boldsymbol{n}$ independent variables:

$$
\begin{aligned}
f(x)= & a_{0}+a_{1} x_{1}+\cdots+a_{n} x_{n}+a_{n+1} x_{1}^{2} \\
& +a_{n+2} x_{1} x_{2}+\cdots+a_{N-1} x_{k}^{m}=\sum_{i=0}^{N-1} a_{i} u_{i}(x)
\end{aligned}
$$

Where $u_{i}(x)$ is the complete polynomial of the design variable $x=\left(x_{1}, x_{2}, \cdots, x_{n}\right)$ in the $\boldsymbol{m}$ power; $a_{i}$ is the undetermined coefficient; The total number of model items is $N=(n+m) ! /(n ! m !) ; \boldsymbol{m}$ is generally set based on the fitting effect of the regression equation, and its value will not exceed 5 .

When $\boldsymbol{m}=\boldsymbol{n}$, the regression model contains the different order interactions of all variables, and the 
model has the strongest ability to explain information, which is usually used to optimize the selection of the model. However, it also leads to the explosive growth of equation terms with the increase of $\boldsymbol{m}$. And the equation, not suitable for the final regression model, is extremely prone to redundant terms. Therefore, it is necessary to prune the equation and select the final regression equation in combination with the engineering meaning and data characteristics.

Considering that it is difficult to directly identify the redundancy of the nonlinear regression equation, a method is proposed to remove redundancy by means of permutation and combination. The calculation process is as follows:

(1) Set $\boldsymbol{m}$ values as $\boldsymbol{n}$-1 and $\boldsymbol{n}$ respectively to calculate complete polynomial adjustment coefficients $\boldsymbol{R}_{n-1}^{2}, \boldsymbol{R}_{n}^{2}$ in the two cases. If $\frac{\left|\boldsymbol{R}_{n-1}^{2}-\boldsymbol{R}_{n}^{2}\right|}{\boldsymbol{R}_{n-1}^{2}} \leq 0.1$, then the complete polynomial in the power of $\boldsymbol{n}-1$ is selected as the initial model; Otherwise, complete polynomials in the $\boldsymbol{n}$ power are chosen. But when $\boldsymbol{R}_{n-1}^{2} \geq 0.9$ the complete polynomial is directly selected to the $\boldsymbol{n}-1$.

(2) Having $\boldsymbol{m}$ been determined in (1), model items $\boldsymbol{N}$ will be fixed. In the next place, according to the combination idea, the adjustment coefficient $\boldsymbol{R}_{k}^{2}$ under different combination of equation terms is calculated when $\boldsymbol{k}$ is set, so the number of models is $\boldsymbol{C}_{N}^{k}=\frac{\boldsymbol{N} !}{\boldsymbol{k} !(\boldsymbol{N}-\boldsymbol{k}) !}$. Then the maximum adjustment coefficient $\max \left(\boldsymbol{R}_{k}^{2}\right)$ of different models will be screened out.

(3) Find minimum $\min \left(\max \left(\boldsymbol{R}_{k}^{2}\right)\right)$ and maximum $\max \left(\max \left(\boldsymbol{R}_{k}^{2}\right)\right)$ value of the maximum adjustment coefficient under different $\boldsymbol{k}\left(\begin{array}{l}\frac{\boldsymbol{N}}{2}, \frac{N}{2}+1, \cdots, N-1, N \text { is even number } \\ \frac{N-1}{2}, \frac{N}{2}+1, \cdots, N-1, N \text { is odd number }\end{array}\right) \cdot$ If
$\frac{\left|\min \left(\max \left(R_{k}^{2}\right)\right)-\max \left(\max \left(R_{k}^{2}\right)\right)\right|}{\min \left(\max \left(\boldsymbol{R}_{k}^{2}\right)\right)} \leq 0.1$,then take the

$\boldsymbol{k} \_$best value corresponding to $\min \left(\max \left(\boldsymbol{R}_{k}^{2}\right)\right)$ as the final number of equation terms to complete the reduction of redundant terms.

(4) When the final term $\boldsymbol{k} \_$best of the equation is determined, the equation with $\max \left(\boldsymbol{R}_{k}^{2}\right)$ is generally selected as the final regression model. However, the actual meaning of the equation should be fully considered in engineering. Therefore, the final term should be determined in combination with physical meaning and data characteristics.

\subsection{Particle swarm algorithm}

Particle swarm algorithm[33, 34]is an excellent optimization algorithm proposed by Eberhart and Kennedy in 1995. Its basic concept originates from the study on the foraging behavior of birds, that is, to find the optimal solution through cooperation and information sharing between different individuals in the population.

First, each particle independently searches for the optimal solution in their own search space, and the optimal solution of each particle will be recorded as the current individual extreme value. In the next step, the optimal solution of the population is determined from different particles, which is also called the current global optimal solution. Finally, according to the current individual extreme value and global optimal solution, the particle search direction and step size will be adjusted continuously until the predetermined goal is reached. The calculation formula is as follows:

$$
\begin{aligned}
v_{i}= & v_{i}+c_{1} \times \operatorname{rand}() \times\left(\text { pbest }_{i}-x_{i}\right) \\
& +c_{2} \times \operatorname{rand}() \times\left(\text { gbest }_{i}-x_{i}\right) \\
x_{i}= & x_{i}+v_{i}
\end{aligned}
$$

Where $\boldsymbol{i}=1,2, \cdots, \boldsymbol{L}, \boldsymbol{L}$ is the total number of particles in the population;

$v_{i}$ is the particle velocity, $\boldsymbol{x}_{i}$ is the current position;

pbest $_{i}$ is the current individual extreme value, gbest $_{i}$ is the current global optimal value;

$c_{1}$ and $c_{2}$ are learning factors respectively, generally a value of 2 ;

However, this standard form of particle swarm algorithm is not stable. In order to improve the reliability, an improved inertial factor algorithm is introduced. The formula is as follows:

$$
\begin{aligned}
v_{i}= & \omega \times v_{i}+c_{1} \times \operatorname{rand}() \times\left(\text { pbest }_{i}-x_{i}\right) \\
& +c_{2} \times \operatorname{rand}() \times\left(\text { gbest }_{i}-x_{i}\right)
\end{aligned}
$$

Where $\omega$ is the inertia factor, and its value is nonnegative. When $\omega$ is large, global optimization ability is strong, but weak in local optimization. On the contrary, global optimization ability is weak with strong local optimization ability.

\section{Results and discussion}

\subsection{Results of sensitivity analysis}

(1) Model test

The iterative target error MSE of BP is set as 0.005 , and $70 \%$ of the samples are divided into training samples and $30 \%$ into test samples. The error convergence process of training set and the relative error of the test set are shown in Fig.4.

As show in Fig. 4, BP model quickly converges to the target error, and the relative error of contact stress in the test set is generally within 0.075 , indicating that the model, used to carry out sensitivity analysis, has good fitting effect and generalization performance and 
strong reliability.

(a) Traing Set

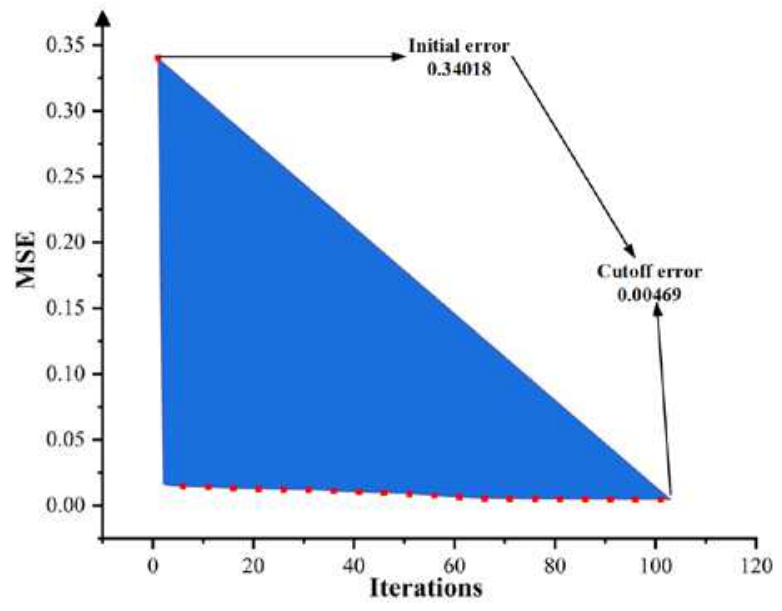

(b) Test Set

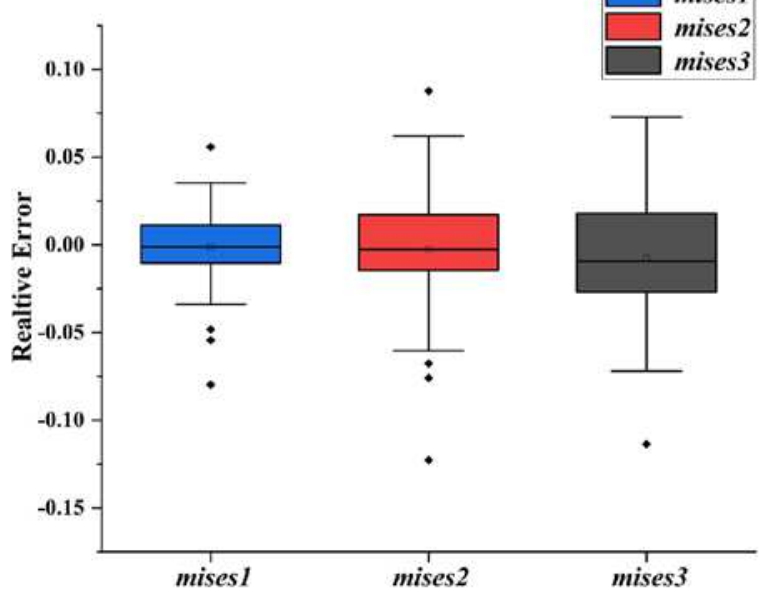

Figure.4 MSE and Relative Error

(2) Sensitivity coefficient

Referring to the research of Song et al[35], set the level parameter $\boldsymbol{p}$ to 10 and disturbance factor $\boldsymbol{m}$ to 0.11 to generate 1000 sets of Morris samples to ensure the reliability of the sensitivity analysis results. And then, due to 264 reconstructed surfaces belonging to large samples, Sobol sensitivity analysis is carried out by the way of utilizing the original samples to better grasp the characteristics of the samples. Sensitivity relative coefficients of $\boldsymbol{S q}, \boldsymbol{S} \boldsymbol{k}$ and $\boldsymbol{S k} \boldsymbol{u}$ are shown in Fig.5.

As can be seen from Fig.5, in the two qualitative and quantitative sensitivity analyses, the order of influence of $\boldsymbol{S q}, \boldsymbol{S} \boldsymbol{s} \boldsymbol{k}$ and $\boldsymbol{S k} \boldsymbol{u}$ under different loads on $\boldsymbol{\sigma}_{\max }$ is $\boldsymbol{S} \boldsymbol{q}>\boldsymbol{S} \boldsymbol{s} \boldsymbol{k}>\boldsymbol{S k} \boldsymbol{u}$, interactively verifying the rationality of the order of parameters.

Table 3. Comprehensive Influence of Parameters

\begin{tabular}{cccc}
\hline Load & $\boldsymbol{S q}$ & $\boldsymbol{S s k}$ & $\boldsymbol{S k u}$ \\
\hline mises1 & 0.8057 & 0.1219 & 0.0724 \\
mises2 & 0.8460 & 0.0898 & 0.0642 \\
mises3 & 0.8689 & 0.0776 & 0.0533 \\
\hline
\end{tabular}

In addition, although height distribution parameters order of Morris and Sobol sensitivity analysis shows consistency in Fig.5, the relative values of the two sensitivity coefficients are still quite different, so it is difficult to compare the influence of height distribution parameters in a same way. In order to observe the comprehensive influence of different height distribution parameters, the relative values of the two sensitivity coefficients are averaged, as shown in Table 3.

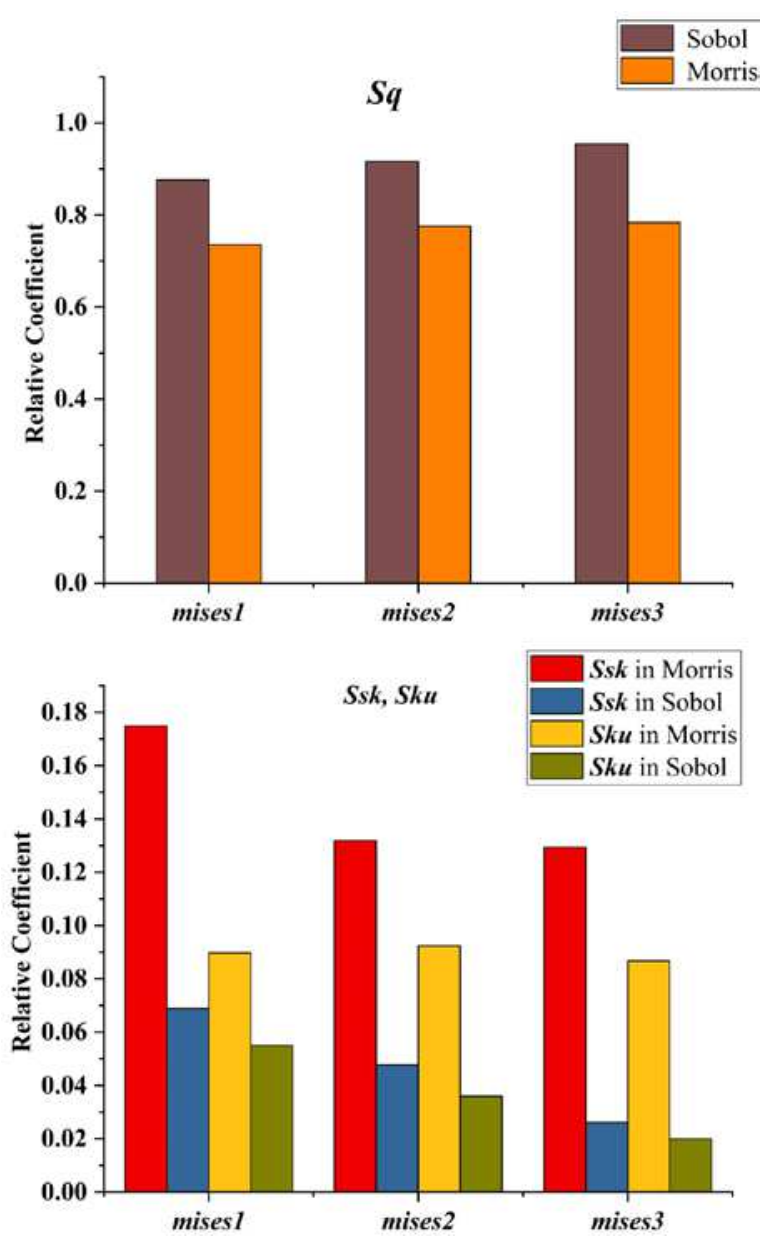

Figure.5 Sensitivity Relative Coefficient

As can be seen from Table 3, when the surface is under light load, the influence of $\boldsymbol{S q}$ reaches $80 \%$, while the influence of $\boldsymbol{S} \boldsymbol{s} \boldsymbol{k}$ and $\boldsymbol{S k \boldsymbol { u }}$ is $20 \%$. However, $\boldsymbol{S} \boldsymbol{s} \boldsymbol{k}$ and Sku will change to about $14 \%$ on heavy load. The results show that with the increase of load, the influence of $\boldsymbol{S s} \boldsymbol{k}$ and $\boldsymbol{S k \boldsymbol { u }}$ decreases gradually, but $\boldsymbol{S q}$ expands continuously. That is, with the increase of the asperities in surface plastic deformation, the surface morphology has changed greatly. The peak areas of asperities are crushed and flattened when they are in contact, the kurtosis of the entire surface is reduced, and the peak characteristics are attenuated. Therefore, the influence of the two on contact stress is significantly reduced, but this does not mean that when surfaces in plastic deformation, the influence of $\boldsymbol{S s} \boldsymbol{k}$ and $\boldsymbol{S k \boldsymbol { u }}$ can be ignored.

It is universally acknowledged that $\boldsymbol{S s} \boldsymbol{k}$ is usually used to describe the surface peak-valley shape, which 
indicates that when $\boldsymbol{S s} \boldsymbol{k}<0$, the surface is generally in a shape of steep valley and round peak, otherwise, it will be sharp peak and round valley. While $\boldsymbol{S k} \boldsymbol{u}$ is used to evaluate the height and depth of peaks and valleys, that is, when $\boldsymbol{S k} \boldsymbol{u}>3$, the surface peaks and valleys are higher and deeper. In sensitivity analysis, Ssk has a stronger influence on contact stress than $\boldsymbol{S k} \boldsymbol{u}$, demonstrating that the peak shape of asperities is more likely to affect contact performance than the volume.

\subsection{Establishment and optimization of the optimal correlation model}

Load and Material parameters, Mech anical perform ance parameters, etc.

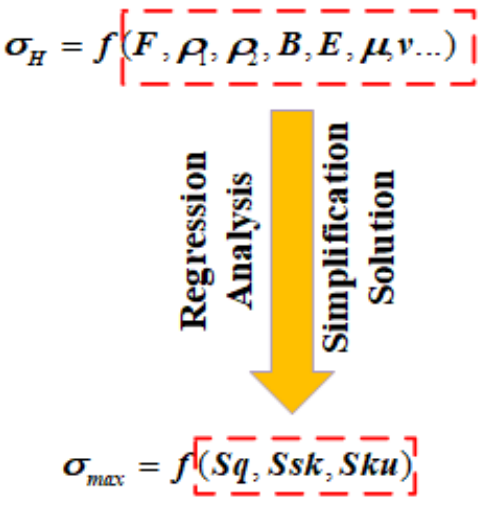

\section{Height distribution parameters}

Figure.6 Regression model

Due to the existence of activation function and node in BP, it is generally not selected as the regression display expression. And considering that the calculation process of rough surface contact stress is complicated and low calculation efficiency, it is necessary to establish a regression model between the height distribution parameter and the maximum mises stress to directly observe the correlation between them. So according to operating conditions, and material performance parameters, nominal contact area and so on, based on the contact model proposed by Wen, the stress sample data is first obtained. With the regression analysis method, many related parameters can be simplified to solve the regression equation coefficients to avoid the lengthy and complicated calculation process. Finally, establish the optimal relationship model in Fig.6 between the height distribution parameters and contact stress.

The analysis is carried out by means of the complete polynomial and optimization method mentioned above. Set the highest degree term to be 2 . The regression model contains the following items, as shown in Table 4.

Complete polynomial regression equations $\boldsymbol{R}^{2}$ under three kinds of loads are calculated:0.9342, 0.9385 and 0.9642 , respectively. Because of the existence of $\boldsymbol{R}^{2}>0.9$, there is no need to solve the complete polynomial under the cubic term. The simplified model is optimized based on the secondorder complete polynomial.

Table 4. Model items

\begin{tabular}{|c|c|c|}
\hline Second Item & Interactive Item & Single Item \\
\hline$S q^{2}$ & SqSsk & $S q$ \\
\hline$S \boldsymbol{s} \boldsymbol{k}^{2}$ & SqSku & Ssk \\
\hline$S k u^{2}$ & SskSku & Sku \\
\hline
\end{tabular}

Table 5 shows the maximum $\boldsymbol{R}^{2}$ of the combination when the model takes different number of items.

Table 5. Maximum $\boldsymbol{R}^{2}$

\begin{tabular}{cccccc}
\hline Load & Four & Five & Six & Seven & Eight \\
\hline mises1 & 0.9268 & 0.9295 & 0.9325 & 0.9328 & 0.9333 \\
mises2 & 0.9363 & 0.9373 & 0.9376 & 0.9378 & 0.9379 \\
mises3 & 0.9584 & 0.9622 & 0.9632 & 0.9637 & 0.9639 \\
\hline
\end{tabular}

The $\min \left(\max \left(\boldsymbol{R}_{\boldsymbol{k}}^{2}\right)\right)$ and $\max \left(\max \left(\boldsymbol{R}_{\boldsymbol{k}}^{2}\right)\right)$ of the maximum adjustment coefficient under different terms are respectively four-term and eight-term, and meet the requirements $\frac{\left|\min \left(\max \left(\boldsymbol{R}_{\boldsymbol{k}}^{2}\right)\right)-\max \left(\max \left(\boldsymbol{R}_{k}^{2}\right)\right)\right|}{\min \left(\max \left(\boldsymbol{R}_{k}^{2}\right)\right)} \leq 0.1$.

Therefore, quadratic four-term should be used as the simplified model. If the quadratic quaternary formula with adjustment coefficient $\max \left(\max \left(\boldsymbol{R}_{k}^{2}\right)\right)$ under different loads is directly selected as the general optimal correlation model at this time, it will be found that the parameter terms under three kinds of loads are different, which does not meet the requirements of the general model and engineering applications.

In order to solve the difference problem of parameter items, the following solutions are proposed:

(1) Under a certain load, select all regression model sets with only thousandths difference from adjustment coefficient $\max \left(\max \left(\boldsymbol{R}_{k}^{2}\right)\right)$. At this point, it can be considered that the models in the regression model sets and the models with $\max \left(\max \left(\boldsymbol{R}_{k}^{2}\right)\right)$ have basically the same explanatory ability to the dependent variables.

(2) Record the frequency of equation different terms in the regression model sets. The higher the frequency of the selected item is, the more likely it is to be the key term to explain the dependent variable, and it is further restricted in combination with the engineering meaning. In this way, the four selected parameter items with the highest frequency can be used as the final regression polynomials under the corresponding load. In this way, it is avoided that some items have a strong explanatory ability for other items, but they happen to catch a "free ride", so that the regression model coefficients also present better fake 
phenomenon.

(3) According to the regression polynomials under different loads, the method in (2) can be used again to unify the parameter differences under multiple loads.

The frequency statistics of parameter items of the three loads are shown in Fig.7.

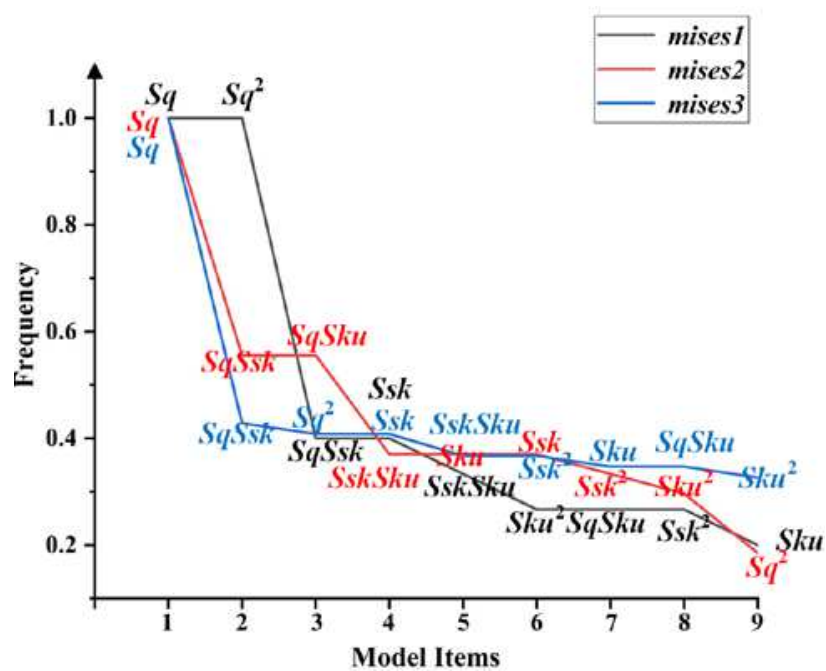

Figure.7 Parameter Frequency

The top four parameter items in mises 1 are $\boldsymbol{S q}$, $S q^{2}, S q S s k, S s k$; mises 2 are $S q, S q S s k, S q S k u, S s k S k u$; mises 3 are $\boldsymbol{S q}, \boldsymbol{S q S s k}, \boldsymbol{S q} \boldsymbol{q}^{2}, \boldsymbol{S} \boldsymbol{s k}$. According to the practical meanning, the equation should contain as much variable information as possible, and there is no Sku item in mises 1 and mises3. Therefore, the fourth item $\mathbf{S k} \boldsymbol{u}$ in mises 1 and mises 3 should be revised, and the fifth item $\boldsymbol{S s} \boldsymbol{k} \boldsymbol{S k} \boldsymbol{u}$ should be used as a replacement. The final optimal relationship model can be determined as follows:

$$
\begin{aligned}
\sigma_{\text {max }}= & b_{0}+b_{1} S q+b_{2} S q^{2}+b_{3} S q S s k \\
& +b_{4} S s k S k u
\end{aligned}
$$

The optimal relationship model is used to solve the regression equation under the three kinds of loads, as shown below:

$$
\begin{aligned}
\text { mises } 1= & 628.681+770.595 S q-542.756 S q^{2} \\
& -2.488 S q S s k+0.206 S s k S k u \quad R_{1}^{2}=0.924 \\
\text { mises } 2= & 762.920+978.235 S q-160.444 S q^{2} \\
& +37.856 S q S s k+0.184 S s k S k u \quad R_{2}^{2}=0.929 \\
\text { mises } 3= & 756.034+2251.518 S q-1079.179 S q^{2} \\
& +111.962 S q S s k+0.025 S s k S k u \quad R_{3}^{2}=0.957
\end{aligned}
$$

The results show that the optimal correlation model fits well under the three loads. Equation adjustment coefficient is only slightly smaller than the maximum adjustment coefficient, and the reliability gets verified.

However, there is still a big gap between engineering surface and reconstruction surface, and the fitting effect of the optimal relationship model on engineering surface height distribution parameters and contact stress is not clear.
In order to clarify the application ability of the optimal relationship model on engineering surface, 264 measured ultrasonic grinding surfaces are selected. Wen's model was used to obtain $\boldsymbol{\sigma}_{\max }$ and verify its fitting effect. Fig. 8 and Fig.9 are the grinding surface diagram an stress sample distribution box figure, respectively.

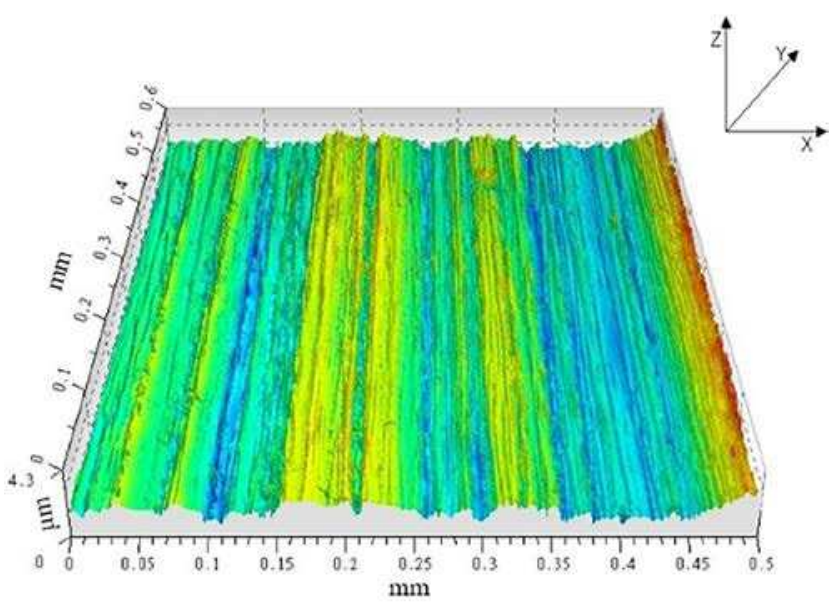

Figure.8 Ultrasonic Grinding Surface

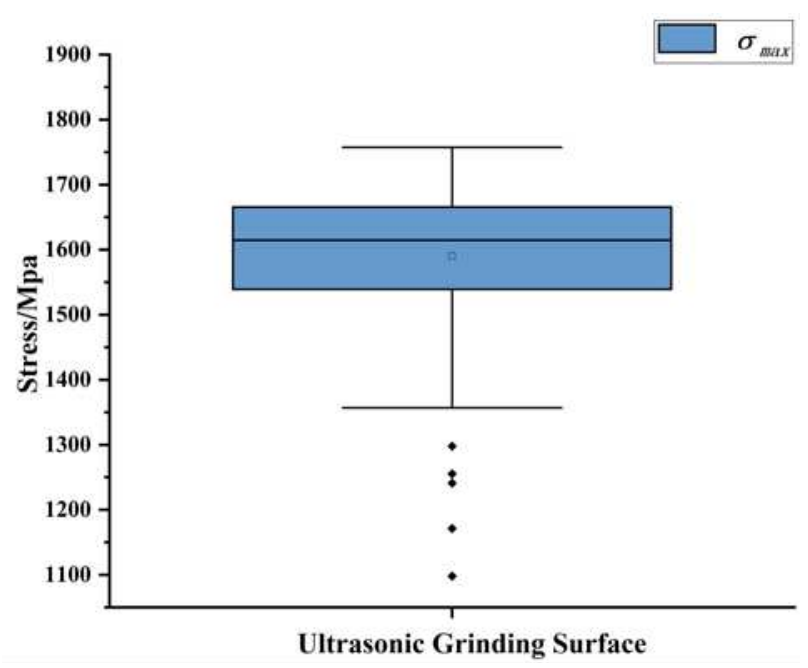

Figure.9 Stress Distribution

The regression equation of ultrasonic grinding surface height distribution parameters and contact stress is shown as follows:

$$
\begin{aligned}
& \sigma_{\max }=909.426+1057.801 S \boldsymbol{q}-353.872 \boldsymbol{S q}^{2} \\
& +39.105 \text { S } \boldsymbol{q} \boldsymbol{S} \boldsymbol{s} \boldsymbol{k}-3.590 \text { SkuSsk } \quad \boldsymbol{R}_{u}^{2}=0.730
\end{aligned}
$$

Under the measured surface, the equation adjustment coefficient $\boldsymbol{R}_{u}^{2}$ is reduced by about 0.2 compared with the reconstruction surface, which seems that the fitting effect of the regression model is not very satisfactory. But the model $\boldsymbol{R}^{2}$ can not fully represent the fitting effect of the nonlinear equation[36, 37], only just for a reference. Therefore, the relative error of ultrasonic grinding surfaces equations should be further observed, as shown in Fig.10.

The model relative error in Fig. 10 is generally less than 0.05 . It proves that the optimal relationship model, 
suitable for predicting contact stress by height distribution parameters under different morphological characteristics, also has excellent fitting ability on the measured surface, further verifying its universality.

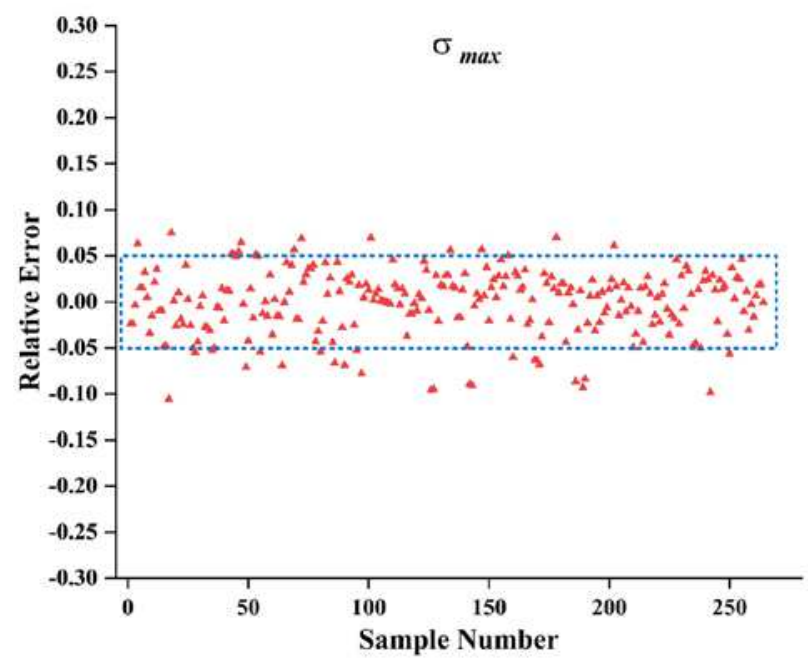

Figure.10 Relative Error

\subsection{Extreme stress value}

Although the optimal relationship model builds the bridge between the height distribution parameters $\sigma_{\text {max }}$, it can only ensure that the equation has sufficient fitting ability while the information of the model will be inevitable to produce partially lost. The optimization algorithm usually requires the model to have a complete information interpretation ability to consider the influence of different factors as much as possible. .

$\boldsymbol{S q}, \boldsymbol{S s k}$ and $\boldsymbol{S k u}$ are the target research objects. Therefore, cubic complete polynomial can fully consider the interaction of the parameters. Meanwhile, cubic polynomial includes the concavity and convexity of the function, meeting the requirements of the basic model of the optimization algorithm.

$$
\begin{aligned}
& f(S q, S s k, S k u)=1497.708 S q+68.284 S k u-531.543 * S s k \\
& -627.862 S q^{2}-103.545 S s k^{2}-13.392 S k u^{2} \\
& +124.294 S q^{3}-1.66 S s k^{3}+0.225 S k u^{3} \\
& +1081.228 * \boldsymbol{S q} * \boldsymbol{S} \boldsymbol{s} \boldsymbol{k}+26.377 \boldsymbol{S q S k u}+2.626 S k u . S s k \\
& -508.453 S q^{2} S s k+9.55 S s k^{2} S q+8.318 S k u^{2} S q \\
& -66.771 S q^{2} S k u+14.682 S s k^{2} S k u+1.425 S k u^{2} S s k \\
& -11.338 \text { SqSskSku+586.712 }
\end{aligned}
$$

The research is carried out with ultrasonic surface. Equation under the cubic complete polynomial is in formula (16).

Then Set $\omega$ to 0.8 , and divide the interval of height distribution parameters based on sample characteristics: $S q[0.6,1.4]$, Ssk[-1.5,1], Sku[2.9,10]. Use particle swarm optimization algorithm to solve the

\begin{tabular}{|c|c|c|c|c|c|c|c|}
\hline \multicolumn{3}{|c|}{ Parameter } & \multirow{2}{*}{$\operatorname{nin}(S p)$} & \multicolumn{2}{|c|}{ Parameter } & \multirow{2}{*}{$\max (\boldsymbol{S p})$} & \multirow{2}{*}{$\begin{array}{l}\text { Impact } \\
\text { Factor }\end{array}$} \\
\hline$S q$ & Ssk & Sku & & Ssk & Sku & & \\
\hline 0.60 & -0.35 & 10 & 1266 & -1.5 & 5.79 & 1436 & 0.119 \\
\hline 0.70 & -0.64 & 10 & 1357 & 1 & 10 & 1492 & 0.090 \\
\hline 0.80 & -1.50 & 2.90 & 1414 & 1 & 10 & 1600 & 0.116 \\
\hline 0.90 & -1.50 & 2.90 & 1441 & 1 & 10 & 1677 & 0.141 \\
\hline 1.00 & -0.08 & 6.09 & 1500 & 1 & 10 & 1725 & 0.131 \\
\hline 1.10 & -1.50 & 2.90 & 1511 & 1 & 10 & 1744 & 0.134 \\
\hline 1.20 & -1.50 & 2.90 & 1556 & 1 & 10 & 1736 & 0.104 \\
\hline 1.30 & -0.30 & 9.72 & 1604 & 1 & 10 & 1700 & 0.057 \\
\hline 1.40 & 1.00 & 6.49 & 1578 & -1.50 & 10 & 1727 & 0.086 \\
\hline
\end{tabular}
minimum $\min (\boldsymbol{S p})$ and maximum $\max (\boldsymbol{S p})$ of $\boldsymbol{\sigma}_{\max }$ under the same $S \boldsymbol{q}$ at an interval of 0.05, and calculate the impact factor $\frac{\max (\boldsymbol{S p})-\min (\boldsymbol{S p})}{\max (\boldsymbol{S p})}$ of $\boldsymbol{S s k}$ and Sku, as shown in Table 6.
As can be seen from Table 6:

Table 6. Extreme Stress

(1) In the case of the same $\boldsymbol{S q}$ but different $\boldsymbol{S s} \boldsymbol{k}$ and $\boldsymbol{S k} \boldsymbol{u}$, the difference between the maximum and minimum of $\sigma_{\max }$ is nearly $250 \mathrm{MPa}$, consistent with the engineering cognition. Otherwise, the influence of $\boldsymbol{S s \boldsymbol { k }}$ and $\boldsymbol{S k} \boldsymbol{u}$ is basically less than $14 \%$, keeping pace with the results of the sensitivity analysis when the surface is in plastic deformation. The accuracy of the model and sensitivity analysis are mutually verified.

(2) In condition that $\boldsymbol{S q}$ is in [0.6,0.7], the minimum $\sigma_{\max }$ is generally generated on the surface with negative $\boldsymbol{S s k}$ but $\boldsymbol{S k} \boldsymbol{u}$ is 10, indicating that surface with strong kurtosis and negative skewness has better contact performance. At this time, the peaks of the surface asperities are rounder and the overall volume is larger, so the bearing capacity gets improved. However, combination of $\boldsymbol{S} \boldsymbol{s} \boldsymbol{k}$ and $\boldsymbol{S} \boldsymbol{k} \boldsymbol{u}$ under the maximum $\boldsymbol{\sigma}_{\max }$ presents great difference with the change of $\boldsymbol{S} \boldsymbol{q}$ but no obvious rule.

(3) Under the circumstances $\boldsymbol{S q}$ is in [0.8,1.2], the minimum $\boldsymbol{\sigma}_{\text {max }}$ is basically unified as $\boldsymbol{S s \boldsymbol { k }}=-1.5$ and $\boldsymbol{S k \boldsymbol { u }}$ =2.9, where surface contact stress $\boldsymbol{S k} \boldsymbol{u}$ close to 3 and negative $\boldsymbol{S s} \boldsymbol{k}$ is smaller. At this time, negative $\boldsymbol{S k \boldsymbol { u }}$ surfaces close to normal distribution have stronger contact performance. The maximum $\sigma_{\max }$ is basically unified as $\boldsymbol{S s} \boldsymbol{k}=1$ and $\boldsymbol{S k u}=10$, which illustrates that kurtosis to 10 and positively skewed surface is in the worst contact performance.

(4) When $\boldsymbol{S q}$ is in the range [1.3,1.4], the rule presented by $\boldsymbol{S s} \boldsymbol{k}$ and $\boldsymbol{S k u}$ combination disappears, and the surface with the maximum and minimum contact stress presents randomness. It indicates that when the surface is too rough, the influence of the surface bearing capacity is no longer the microscopic morphology characteristics but the macroscopic shape characteristics, and the microscopic morphology characteristics lose their role in regulating the contact performance.

\section{Conclusion}

(1) With the help of BP neural network and global 
(qualitative and quantitative) sensitivity analysis method, the influence of height distribution parameters on contact stress gets studied. Under different loads, the order of contact stress is consistent in $\boldsymbol{S q}>\boldsymbol{S} \boldsymbol{s} \boldsymbol{>} \boldsymbol{S k} \boldsymbol{u}$. And with the increase of load, the influence of $\boldsymbol{S} \boldsymbol{s} \boldsymbol{k}$ and $S \boldsymbol{k} \boldsymbol{u}$ gradually decreases. When the surface is in elastic deformation, the influence of $\boldsymbol{S q}$ is $80 \%$, but $\boldsymbol{S s \boldsymbol { k }}$ and $\boldsymbol{S k \boldsymbol { u }}$ is $20 \%$. The influence of $\boldsymbol{S} \boldsymbol{s}$ and $\boldsymbol{S k \boldsymbol { u }}$ on surface plastic deformation caused by heavy load contact is about $14 \%$.

(2) Based on the idea of complete polynomials and combination, and combined with data characteristics and engineering physics significance, a method of optimizing and reducing complete polynomials is proposed. An optimal relationship model between height distribution parameters and contact stress is established by means of the method: $\sigma_{\text {max }}=b_{0}+b_{1} S q+b_{2} S q^{2}+b_{3} S q S s k$.The model shows $+b_{4} S s k S k u$

good fitting performance and strong reliability in different surface and multi-load contact analysis.

(3) According to the particle swarm optimization algorithm, the extreme value of surface contact stress under different $\boldsymbol{S q}$ is solved. The results show that when $\boldsymbol{S q}$ is in a lower value, surface contact performance with negative strong kurtosis is stronger. As the surface becomes rougher, the contact performance of the negatively skewed surface close to the normal distribution is better. However, when the surface is too rough (surface is more prone to plastic deformation under the same load), it indicates that the influence of microscopic morphology on the surface bearing capacity is low but mainly controlled by the macroscopic shape characteristics.

\section{Acknowledgment}

The author(s) disclosed receipt of the following financial support for the research, authorship, and/or publication of this article: National Key R\&D Program of China (Grant No.2018YFB2001300), National Natural Science Foundation of China (NSFC) through Grant No. 51705142.

\section{Conflict of Interest}

There are no conflicts of interest.

\section{Reference}

[1] Suh, A.Y., Polycarpou, A.A., Conry, T.F. Detailed surface roughness characterization of engineering surfaces undergoing tribological testing leading to scuffing. Wear 255:556-568 (2003).

[2] Jadhav, S., Thakre, G.D., Sharma, S.C. An experimental study on tribological performance and surface characteristics of lubricated point contact. Acta Mechanica 229:4413-4430 (2018).

[3] Singh, R., Melkote, S.N., Hashimoto, F. Frictional response of precision finished surfaces in pure sliding. Wear 258:1500-1509 (2005).

[4] Magsipoc, E., Zhao, Q., Grasselli, G.: 2D and 3D roughness characterization. Springer, Place Springer (2020)

[5] Whitehouse, D.J. Surface characterization and roughness measurement in engineering. Photomechanics:413-461 (2000).

[6] XIAO, H., WANG, X.-z., XIE, T.-b. A Measuring Instrument for Curved Surface Profile of Rolling Bearings. Bearing (2004).

[7] De Chiffre, L., Lonardo, P., Trumpold, H., Lucca, D.A., Goch, G., Brown, C.A., et al. Quantitative characterisation of surface texture. CIRP Annals 49:635-652 (2000).

[8] Shimizu, M., Sawano, H., Yoshioka, H., Shinno, H.: Surface texture characterization of ultra-precision machined parts based on laser speckle pattern analysis. 12th International Conference of the European Society for Precision Engineering and Nanotechnology, EUSPEN 2012, pp. 154-157 \% 2021-2006-2025 2016:2053:2000. euspen, Place euspen (2012)

[9] Deltombe, R., Kubiak, K.J., Bigerelle, M. How to select the most relevant $3 \mathrm{D}$ roughness parameters of a surface. Scanning: The Journal of Scanning Microscopies 36:150-160 (2014).

[10] ISO, D. Geometrical Product Specifications (GPS)_-surface texture: profile method-terms, definitions and surface texture parameters. 4287: 1997/Cor1: 1998 (1997).

[11] Clausen, R., Stangenberg, J.: Roughness of shotpeened surfaces-definition and measurement. Proceedings of the 7 th international conference on shot peening, pp. 69-77. Place (1999)

[12] Standardization, I.O.F. ISO 25178-2: 2012: geometrical product specifications (GPS)-Surface texture: areal-Part 2: terms, definitions and surface texture parameters. International Organization for Standardization (2012).

[13] He B, Wei C, Liu B, et al. Three-dimensional surface roughness characterization[J]. Optics and Precision Engineering. 2018, 26(08): 1994-2011.

[14] Dong, W.P., Sullivan, P.J., Stout, K.J. Comprehensive study of parameters for characterising 
three-dimensional surface topography: IV: Parameters for characterising spatial and hybrid properties. Wear 178:45-60 (1994).

[15] Zeng, Q., Qin, Y., Chang, W., Luo, X. Correlating and evaluating the functionality-related properties with surface texture parameters and specific characteristics of machined components. International Journal of Mechanical Sciences 149:62-72 (2018).

[16] Tayebi, N., Polycarpou, A.A. Modeling the effect of skewness and kurtosis on the static friction coefficient of rough surfaces. Tribology international 37:491-505 (2004).

[17] Sedlaček, M., Podgornik, B., Vižintin, J. Correlation between standard roughness parameters skewness and kurtosis and tribological behaviour of contact surfaces. Tribology international 48:102-112 (2012).

[18] Sedlaček, M., Podgornik, B., Vižintin, J. Planning surface texturing for reduced friction in lubricated sliding using surface roughness parameters skewness and kurtosis. Proceedings of the Institution of Mechanical Engineers, Part J: Journal of Engineering Tribology 226:661-667 (2012).

[19] Yan, X.-L., Wang, X.-L., Zhang, Y.-Y. Influence of roughness parameters skewness and kurtosis on fatigue life under mixed elastohydrodynamic lubrication point contacts. Journal of Tribology 136 (2014).

[20] Zhu, S., Huang, P. Influence mechanism of morphological parameters on tribological behaviors based on bearing ratio curve. Tribology International 109:10-18 (2017).

[21] Duo, Y., Jinyuan, T., Wei, Z., Yuqin, W. Correlation between surface roughness parameters and contact stress of gear. Proceedings of the Institution of Mechanical Engineers, Part J: Journal of Engineering Tribology 235:551-563 (2021).

[22] Duo, Y., Jinyuan, T., Wei, Z., Yuqin, W. Study on Roughness Parameters Screening and Characterizing Surface Contact Performance Based on Sensitivity Analysis. Journal of Tribology 144:041502 (2021).

[23] Liao, D., Shao, W., Tang, J., Li, J. An improved rough surface modeling method based on linear transformation technique. Tribology International 119:786-794 (2018).

[24] Liao, D., Shao, W., Tang, J., Li, J., Tao, X. Numerical generation of grinding wheel surfaces based on time series method. The International Journal of
Advanced Manufacturing Technology 94:561-569 (2018).

[25] Wen, Y., Tang, J., Zhou, W., Li, L., Zhu, C. New analytical model of elastic-plastic contact for threedimensional rough surfaces considering interaction of asperities. Friction:1-15 (2021).

[26] Lipiński, D., Bałasz, B. Modelling of surface roughness and grinding forces using artificial neural networks with assessment of the ability to data generalisation. The International Journal of Advanced Manufacturing Technology 94:1335-1347 (2018).

[27] Morris, M.D. Factorial sampling plans for preliminary computational experiments. Technometrics 33:161-174 (1991).

[28] Campolongo, F., Cariboni, J., Saltelli, A. An effective screening design for sensitivity analysis of large models. Environmental modelling \& software 22:1509-1518 (2007).

[29] Zadeh, F.K., Nossent, J., Sarrazin, F., Pianosi, F., van Griensven, A., Wagener, T., et al. Comparison of variance-based and moment-independent global sensitivity analysis approaches by application to the SWAT model. Environmental Modelling \& Software 91:210-222 (2017).

[30] Sobol', I.M., Asotsky, D., Kreinin, A., Kucherenko, S. Construction and comparison of high-dimensional Sobol'generators. Wilmott 2011:64-79 (2011).

[31] Chen, R., Han, X., Liu, J., Zhang, W. A computational inverse technique to determine the dynamic constitutive model parameters of concrete. Computers, Materials, \& Continua 25:135-157 (2011). [32] Kai, Y., Chen, S., Zheng, B., Zhang, K., Yang, N., $\mathrm{Xu}, \mathrm{W}$. Qualitative and quantitative analysis of nonlinear dynamics by the complete discrimination system for polynomial method. Chaos, Solitons \& Fractals 141:110314 (2020).

[33] Poli, R., Kennedy, J., Blackwell, T. Particle swarm optimization. Swarm intelligence 1:33-57 (2007).

[34] Wang, F., Zhang, H., Zhou, A. A particle swarm optimization algorithm for mixed-variable optimization problems. Swarm and Evolutionary Computation 60:100808 (2021).

[35] Song X, Kong F, Zhan C, et al. Sensitivity analysis of hydrological model parameters using a statistical theory approach[J]. Advances in Water Science. 2012, 23(5): 642-649.

[36] Spiess, A.-N., Neumeyer, N. An evaluation of R 2 
as an inadequate measure for nonlinear models in pharmacological and biochemical research: a Monte Carlo approach. BMC pharmacology 10:1-11 (2010).

[37] Sapra, R.L. Using R2 with caution. Current Medicine Research and Practice 4:130-134 (2014). 